\title{
Efficacy of rehabilitation interventions in rheumatic conditions
}

\section{Gerold Stucki, MD, and Matthew H. Liang, MD, MPH}

\author{
Brigham and Women's Hospital, Boston, Massachusetts, USA
}

\begin{abstract}
All industrialized nations are facing a crisis in health care financing. Rising expectations coupled with increasing specialization and technologic capacities have forced health care payers to examine their assumptions and to seek data on the outcomes of medical interventions. Clinical investigators who have been taught to use randomized controlled trials that evaluate efficacy under experimental conditions have been redirected toward studies that can help answer health policy questions. Such studies examine the effectiveness of interventions in more realistic settings on a richer array of patient-centered outcomes such as function and consider cost effectiveness and relative cost effectiveness. Rehabilitation interventions, which are by and large pragmatic, have never had a strong scientific basis grounded in controlled trials, and this lack of evidence has put tremendous pressure on clinicians to justify their practices. In this article, we review the recent literature on effectiveness of rehabilitation interventions in rheumatic disorders.
\end{abstract}

Current Opinion in Rheumatology 1994, 6:153-158

\section{Nonarticular rheumatism}

Disorders collectively called nonarticular rbeumatism may be the most important rheumatic conditions from a public health standpoint. In a study from Addenbrooke's Hospital in Cambridge, the authors critically tested the efficacy of laser treatment of rotator cuff tendinitis $\left[1^{\bullet \bullet}\right]$. The condition was defined by the criteria of Cyriax as shoulder pain with a painful arc at abduction between $40^{\circ}$ and $120^{\circ}$ and a painful resisted movement in at least one range of motion abduction, external or internal rotation. Patients with frozen shoulder, acromioclavicular arthritis, or rotator cuff tears were excluded, as were patients who were pregnant, breast feeding, or who had received intra-articular or subacromial steroids in the 3 months prior to treatment. $\mathrm{Pa}$ tients with systemic rheumatic illnesses or patients who had received physical therapy were also excluded. Current anti-inflammatory therapy was stopped, and a week of washout was allowed before baseline assessments were made. Range of motion, painful arc score, resisted movement score, and pain at night, during rest, and at movement was measured weekly by visual analogue scales and functional limitation. Thirty-five patients were randomized to receive treatment with an active low-level laser emitting a continuous $30 \mathrm{MW}$ beam with a wavelength of $830 \mathrm{~nm}$ or dummy laser treatment twice weekly for 8 weeks. Paracetamol was allowed as needed up to $2 \mathrm{~g} / \mathrm{d}$. All of the measures improved from baseline, but there was no difference between the two groups at any point, and the authors concluded that laser treatment is not more effective than placebo.

Stretching, mobilization, and manipulation are related techniques used by physical therapists and chiropractors in the treatment of patients with low back pain. Few controlled studies have evaluated their efficacy, and the way in which manipulation, mobilization, and stretching are used vary considerably. In a study from the University of Miami, the authors examined stretching in a subset of patients with chronic low back pain due to myofascial syndrome [2]. Persons with no low back pain or evidence of radiculopathy or nonmuscular-related organic disorders or other medical causes of low back pain were excluded from the trial. The program consisted of four sessions of stretching over 2 weeks; a comparison group participated in an identical program without the stretching sessions. All patients were seen in a multidisciplinary low back pain rehabilitation center and received therapeutic exercises, muscle strengthening, heat, ice, ultrasound, traction, progressive resistant exercises, and other therapy in addition to the stretching that was evaluated. 
The principal outcome measures included pain, static strength of the back extensors measured by standardized pulling tests, and lumbar paraspinal muscle activity measured by electromyography. Patients were evaluated before treatment and at the end of the 2 weeks.

Of 28 persons, patients undergoing the rehabilitation program with and without systemic stretching showed significant improvement in strength and, more importantly, in pain. This study is of interest but is limited by its statistical analysis, the inability to separate the effect of the other components of the rehabilitation program from stretching, and the lack of a true comparison to the stretching maneuver such as sham stretching.

\section{Osteoarthritis}

\section{Behavioral approaches}

A major component of comprehensive rehabilitation is the use of psychoeducational techniques to reduce pain and psychological distress and improve function. In the most experienced patients, ie, those followed by rheumatologists, planned psychoeducational interventions improve symptoms above what is achieved with medications alone.

In a study from the University of California, San Diego, the authors evaluated cognitive-behavioral modification in patients with osteoarthritis [3]. Forty adults with unspecified forms of osteoarthritis were randomized to receive either cognitive-behavioral modification or education alone. The group who received cognitivebehavioral modification was taught to reconceptualize their pain, monitor their thoughts, feelings, and actions and the relationship between them, and to strengthen their belief that they can cope effectively. The patients also received help in making behavioral changes to improve functioning. Techniques included cognitive reappraisal, imagery, relaxation training, mental distraction, and goal setting. The control group received lectures presented by a physician or an allied health professional on information generally believed to be useful for patients with osteoarthritis. The two groups were interviewed by a blinded assessor focused on the quality of well-being, the Arthritis Impact Measurement Scales (AIMS), the Beck Depression Inventory, and the Social Support Questionnaire. These interviews took place at 2,6 , and 12 months following intervention. At baseline, the two groups were comparable, but in the group receiving cognitive-behavioral intervention, $15 \%$ of the patients had osteoarthritis for less than five years compared with the educational group, in which there were no patients who had osteoarthritis for less than 5 years. Twenty-five percent of the patients dropped out or were lost to 1-year followup. Pretest measures for those who dropped out or for whom data were missing were compared with the patients who remained in the study; $75 \%$ of the pretest scores significantly differed from those of the patients who stayed in the study. By 1 year, there was no difference in physical or psychological functioning between the groups. Although the authors concluded that cognitive behavior modification was no more effective than nonspecific education about arthritis, one cannot be sure from the results of this study alone. Results of studies that show no effect might be due to no effect, insensitive measures, inadequate sample size, bias, or dropouts, all of which could have been factors in this study.

\section{Physical modalities}

Osteoarthritis commonly causes limited knee mobility; loss of $10^{\circ}$ of extension can alter biomechanics of the hip and ankle, which increases energy expenditure, dysfunction, and pain. In osteoarthritis, there is reduced elasticity of capsular soft tissue. Ultrasound has been used to enhance connective tissue extensibility, and in uncontrolled, unblinded trials, it increased joint motion in osteoarthritis. In a 2-month randomized, double-blind controlled trial, ultrasound with exercise was compared with exercise alone in 79 patients with chronic knee osteoarthritis and impaired mobility [ $\left.4^{\bullet}\right]$. Overall, there were no significant differences in active range of motion or pain between the groups, although both groups improved over baseline in these parameters. The lack of effect may be due to the marginal effect of ultrasound compared with that of exercise, small sample size, insensitive or imprecise measures, or the dose or site of delivery of ultrasound. Nevertheless, ultrasound appears to add little to exercise in improving mobility or reducing pain.

In a Canadian study, the authors undertook a 6-week double-blind, randomized placebo-controlled trial of transcutaneous electrical nerve stimulation with random switching between electrodes to avoid habituation in patients with osteoarthritis of the hip or knee [5]. The placebo group received sham therapy with a low frequency that could be felt by sensitive individuals, and the experimental group was encouraged to use the highest intensity that could be tolerated without inducing pain. Acupuncture points were used to stimulate the supraspinal, sciatic, perineal, femoral, sural, saphenous, and tibial nerves. The initial two therapies were performed in the clinic, and patients were then asked to use the device twice daily for 30 minutes at predetermined points. Sixty-six patients started the study, but 19 were excluded at the beginning or subsequently for noncompliance. Patients were evaluated by rheumatologists before treatment and at 6 weeks for functional status, range of motion of the involved hip or knee, knee circumference, 50-foot walk time, and tenderness of the involved joint and soft tissue. Pain was measured by visual analogue scale and the West Haven Yale Multidimensional Pain Inventory, which were the only scores that improved by $25 \%$ or more in the treated compared with the sham group. Although the study was controlled and randomized, there is not 
enough information presented to determine the rigor of the evaluation. This includes rudimentary information on diagnostic criteria, criteria for inclusion-exclusion, cotherapies, and success of randomization. It is likely that the sham and active interventions were not administered identically, and most importantly, no primary data are presented.

\section{Therapeutic exercise}

The role of weight-bearing exercise in early osteoarthritis of the knee is debated and is reflected by advice to patients recommending everything from limiting walking to using walking as therapy. In a welldone randomized, controlled trial, supervised fitness walking and patient education were compared with usual medical care over 2 months [6*0]. One hundred two patients over 40 years of age with radiographic osteoarthritis and one or both knees symptomatic on weight-bearing for at least 4 months were included. The program comprised 2490 -minute sessions in a hospital, of which up to 30 minutes per session were spent walking. Intervention included light stretching and strengthening exercises, education on medical aspects of osteoarthritis and exercise, group discussions about benefits and barriers of walking, and instruction in proper walking and maintenance of a walking program. Patients who underwent the walking program showed an $18 \%$ improvement in timed walking ability, a $39 \%$ improvement on the AIMS physical activity subscale, $27 \%$ less pain, and less frequent pain medication use. The group receiving usual treatment had decreased walking ability from baseline, did not improve on the AIMS physical activity subscale, and used more medications. These effects occurred without exacerbating arthritis-related symptoms. Whether the positive effects can be maintained over a longer period of time is the subject of an ongoing study that will provide much needed information on the usefulness and cost effectiveness of the program.

\section{Rheumatoid arthritis}

\section{Education}

The quality of life and the course of rheumatoid arthritis can be influenced by educational strategies that may

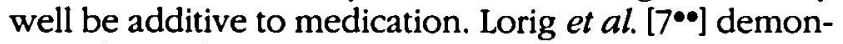
strated in a short-term controlled study that the Arthritis Self-Management Program (ASMP) achieves reductions in pain and depression together with increased physical activity, although no change in disability was seen. In an observational follow-up after 4 years of 224 (79\%) of 284 patients who completed the ASMP, $20 \%$ less pain, $40 \%$ fewer physician visits, and a $9 \%$ improvement in physical disability were seen. Calculated savings per patient for the 4-year period due to reduced use of resources were estimated to be $\$ 648$, which compares favorably with the total expenditure of $\$ 54$ for the ASMP per participant. A nonrandom concurrent comparison group derived from an observational study on volunteers in the same geographic region during the same time period experienced worsening of pain and physical disability and an increased number of physician visits. Although the results of this study need to be confirmed, they are encouraging, and one could argue that health education programs should be adopted for economic reasons alone because they are likely to be cost saving.

\section{Therapeutic exercise}

Exercise has been shown to be effective in preventing the negative health effects due to deconditioning and may be useful in preventing osteoporosis in patients with rheumatoid arthritis. However, there is no agreement on what type of exercise, $i e$, isometric exercises, stretching, coordination, or endurance, should be prescribed. The inclusion of weight-bearing exercises is still debated but may be important to maintain bone density. Kirsteins et al. [8] suggest that the traditional Chinese exercise tai-cbi chuan has all these qualities and can be practiced safely by patients with rheumatoid arthritis when some modifications such as limited knee flexion are adopted. Forty-seven graduates of the arthritis self-help class were randomized to receive taichi chuan or to a control group doing usual activities. No increase in disease activity compared with that of the controls was observed. Apart from soreness of the knee, shoulder, and lower back during the first weeks of the 10-week study period, occurring particularly after the 90-minute instruction given once a week, no side effects were recorded. Improvement in symptoms, activities of daily living, and general well being were not quantified. Although the long-term effects on general well being, symptoms, posture, function, aerobic and cardiovascular capacity, and bone density need to be established, this exercise is an interesting alternative to the often monotonous traditional programs. When patients can choose among various useful and safe exercise programs they are likely to better adhere to the treatment regimen.

\section{Physical modalities}

The application of heat in rheumatoid arthritis has a long tradition as adjunct therapy but has undergone only limited study of scientific rigor. Sukenik et al. [9] undertook a randomized, double-blinded study in 28 patients on the efficacy of mud packs heated to $40^{\circ} \mathrm{C}$ applied over the four extremities, abdomen, and back for 20 minutes. Patients in the experimental group had reduced morning stiffness, reduced joint counts, and increased grip strength for up to 3 months. Patients in the control group who received washed-out mud packs did not show these effects. No disease flares occurred in either group. Although it would have been crucial, no data on disease activity were provided. Based on the trial, one therefore cannot answer the question of to whom this active heat therapy might be 
applied safely. Why heat did not result in improvement in the control group and whether the suggested absorption of zinc explains the observed difference remains debatable.

\section{Rehabilitation of the rheumatoid hand}

Hand function is critical in patients with rheumatoid arthritis. Rehabilitation efforts are probably most beneficial in early disease. Hand splinting traditionally has been used for acutely inflamed joints to reduce inflammation, pain, and morning stiffness, but compliance is problematic. To increase compliance with splint use, Feinberg [ $\left.10^{\circ}\right]$ used learning principles, sharing of expectations, affective tone and behaviors by the therapists, and assumption of responsibility by the patient. The intervention group recorded the use of the splint for at least 3 hours per day for an average of 23.3 days, which was significantly different from an average of 18.1 days in the standard-therapy group, using nonparametric statistics. Change in the amount of wrist and hand pain was not significant in either group; however, the intervention group experienced a decrease in the duration of morning stiffness. These principles seem generalizable to other splint prescriptions, as well.

An alternative to splinting the inflamed rheumatoid hand is the use of compression gloves. In a randomized controlled trial, McKnight and Kwoh [11'] compared the relative efficacy of two commercially available compression gloves for nighttime treatment. Because the efficacy of compression gloves has been shown in previous studies, no control group was used. Both gloves proved equally effective in reducing pain, morning stiffness, swelling of the proximal interphalangeal joints, and in increasing range of motion (ROM), finger motion, and grip strength. However, because the patients participated in an in-hospital rehabilitation program during the 7-day study period, the treatment effects cannot be separated from those of other treatment. Patients with carpal tunnel syndrome experienced worsening of their symptoms, which underlines the need for careful selection and monitoring of patients even for "simple" measures. An interesting finding was that patient preferences for the two types of gloves varied. Some patients did not like the wool glove, which they described as scratchy and hot, whereas others did not like the nylon glove because it was difficult to apply. Addressing patient preferences when prescribing devices is important in improving compliance.

Whereas resting the hand may reduce symptoms, ROM and strengthening exercises are believed to be important in maintaining cartilage nutrition and function. However, the effectiveness and specifics of exercise programs for the rheumatoid hand are controversial.

In a study of the effectiveness of hand exercise in improving hand strength, Hoenig et al. [12] randomized
57 patients to receive either ROM exercises (for fingers and thumb), balanced resistive exercises (finger abduction and adduction and gross grip using therapeutic putty 85 ), a combination of both treatments, or no treatment. Because of the small sample size and multiple comparisons, the results should be considered preliminary, especially with respect to secondary outcomes such as ROM, pain, deformities, hand disease activity, and dexterity. By comparing the pooled data from all three intervention groups with that of the controls, the authors found a significant difference in strength for the left hand (change from 72.1 to 89.3 $\mathrm{mm} \mathrm{Hg}$ vs 83 to $81.1 \mathrm{~mm} \mathrm{Hg}$ in the control group). However, from the data given, right hand strength improved in the control group (from 68.2 to $81.1 \mathrm{~mm} \mathrm{Hg}$ ) as much as in the pooled treatment groups (from 83.1 to $90 \mathrm{~mm} \mathrm{Hg}$ ). Significantly more patients in the resistive exercise groups reported some problem with the upper extremities and decreased the frequency of sessions and/or number of repetitions per session.

Dellhag et al. [13] randomized 52 patients to receive either exercise (avoiding ulnar deviation of fingers), waxbath treatment, a combination of both, or no treatment. Exercise treatment was given in a group three times per week for 4 weeks in an occupational therapy department and took about 20 minutes for each session. Wax-bath treatment had no beneficial effect except for short-term pain relief (data were not shown, however). The results need to be interpreted with caution because of small sample sizes and multiple comparisons. The data presented do not support the authors' claim that wax-bath treatment seems to facilitate exercise to improve hand function more with combined treatment vs. simple exercise. One also wonders whether the significant decrease of pain during nonresisted motion and stiffness for the exercise group reflect true differences or chance.

Table 1. Home exercise program for the rheumatoid hand"
Warm up, fast flexion, and extension of the fingers 10 times
Place hands flat on table and try to lift (extend) each finger off a
table 5 times
Roll and unroll a crepe bandage 5 times
Roll and unroll a bath towel 5 times
Grip a sheet of paper between thumb and each finger alternatively and
try to pull the paper out; repeat with both hands 5 times
Repeatedly flex and extend metacarpophalangeal joints by placing the
hands over the edge of a table at the level of the
metacarpophalangeal joints; in a similar fashion the proximal
interphalangeal joints are also exercised

In the only long-term study on the effectiveness of an exercise program, Brighton et al. [14*0] randomized 44 patients with seropositive rheumatoid arthritis to a specified daily exercise program (Table 1) and to a control group not given any exercises. At the end of 4 years, there was a significant improvement in grip 
strength and pincer grip strength in the experimental group, whereas the control group showed a significant deterioration. Extensive follow-ups with assessment of compliance and motivational reinforcements as needed were done at least every 6 months and at every physician visit. With respect to joint motion, the metacarpophalangeal joints deteriorated significantly in both groups, but much less in the treatment group.

These studies suggest that hand strength may be maintained or improved with regular exercise. When carefully monitored and reinforced, home exercise may be a cost-effective alternative to group therapy. No conclusions with regard to reduction of local disease activity and avoidance of deformity can be drawn. Future studies should measure adequate sample sizes for specific tests of hypothesis. Whether exercise programs improve hand function and performance of activities of daily living needs further study. The use of preparing physical therapy such as mild moist packs needs to be studied because the effect of the hot wax bath in easing exercise could not been demonstrated.

\section{Perioperative rehabilitation}

Continuous passive motion (CPM) is used commonly for postoperative rehabilitation of patients who have undergone knee surgery. Two randomized controlled trials independently demonstrated the effectiveness of continuous passive motion in patients undergoing total knee arthroplasty. Johnson et al. [15'] compared CPM with splinting for 7 days; they observed earlier discharge and better ROM at 12 months in the experimental group. In the first blinded study to date, McInnes et al. $\left[16^{\bullet \bullet}\right]$ compared the standard treatment program with a combination of the standard program and CPM. Use of CPM increased active flexion and decreased swelling, whereas pain, active and passive extension, and quadriceps strength were not significantly different from those of the control group. In the experimental group, none of the 45 patients required manipulation, whereas eight of the 44 patients in the standard rehabilitation group required manipulation for unsatisfactory recovery of ROM. The costs of manipulative treatments were higher than the additional costs due to use of CPM, resulting in cost savings with the experimental treatment despite a nonsignificant difference with respect to length of stay. In both studies, no increased frequency of wound healing problems was seen with the use of CPM. Whether a standardized protocol increasing range of motion on a predefined basis and use of CPM for up to 20 hours [15\%] is superior to increasing the range of motion daily as tolerated and encouraging patients to use CPM "as much as possible" [16॰日] remains open to debate.

\section{Conclusions}

The results of controlled trials have confirmed new interventions and, of equal importance, have demonstrated the lack of or marginal effectiveness of widely used but scientifically unproven clinical practices. Future research may address the problem of the additional benefit of specific treatments, the value of multiple interventions that are usually used in comprehensive rehabilitation, and the question of what treatment is most effective for whom.

\section{References and recommended reading}

Papers of particular interest, published within the annual period of review, have been highlighted as:

- Of special interest

-. Of outstanding interest

1. Vecchio P, CAVE M, KING V, ADEBajo AO, SMITH $M$, - Hazleman BL: A Double-Blind Study of the Effectiveness of Low Level Laser Treatment of Rotator Cuff Tendinitis. Br J Rbeumatol 1993, 32:740-742.

In this carefully conducted randomized trial, laser treatment was not significantly better than dummy laser treatment with respect to range of motion, painful arc score, resisted movement score, pain at night, pain at rest, and functional limitation

2. KHALIL TM, ASFOUR SS, MARTINEZ LM, WALY SM, ROSOMOFF RS, ROSOMOFF HL: Stretching in the Rehabilitation of LowBack Pain Patients. Spine 1992, 17:311-317.

3. CALFAS KJ, KAPLAN RM, INGRAM RE: One-Year Evaluation of Cognitive-Behavioral Intervention in Osteoarthritis. Arthritis Care Res 1992, 5:202-209.

4. FALCONER J, HAYES KW, CHANG RW: Effect of Ultrasound - on Mobility in Osteoarthritis of the Knee. Arthritis Care Res 1992, 5:29-35.

In a 2-month randomized double-blind controlled trial, ultrasound therapy added no significant effect to an exercise program with respect to pain and active range of motion.

5. FARGAS-BABjaK AM, POMERANZ B, ROONEY PJ: AcupunctureLike Stimulation With Codetron for Rehabilitation of Patients With Chronic Pain Syndrome and Osteoarthritis. Acupunct Electrotber Res 1992, 17:95-105.

6. Kovar PA, Allegrante JP, MacKenzie CR, Peterson MGE, - GUTTN B, ChARISON ME: Supervised Fitness Walking in Patients With Osteoarthritis of the Knee. Ann Intern Med 1992, 116:529-534.

A program of stretching, strengthening, education, group discussions, and supervised walking improved timed walking capacity, decreased pain, and increased physical activity.

7. LORIG KR, MAZONSON PD, HOLMAN HR: Evidence Sug-. gesting That Health Education for Self-Management in Patients With Chronic Arthritis Has Sustained Health Benefits While Reducing Health Care Costs. Artbritis Rbeum 1993, 36:439-446.

This study provides evidence that health education in chronic arthritis adds significant and sustained benefits to medical therapy while reducing health care utilization.

8. KIRSTEINS AE, DIETZ F, HwANG SM: Evaluating the Safety and Potential Use of a Weight-Bearing Exercise, Tai-Chi Chuan, for Rheumatoid Arthritis Patients. Am J Phys Med Rebabil 1991, 70:136-141.

9. SUKENIK S, BUSKILA D, NEUMANN L, KLEINER-BAUMgarten A: Mud Pack Therapy in Rheumatoid Arthritis. Clin Rbeumatol 1992, 11:243-247. 
10. FEINBERG J: Effect of the Arthritis Health Professional on - Compliance With Use of Resting Hand Splints by Patients With Rheumatoid Arthritis. Arthritis Care Res 1992, 5:17-23. The use of learning principles, sharing of expectations, affective tone and behaviors by the therapists and the assumption of responsibility by the patient may increase patient compliance for wearing a resting hand splint.

11. MCKNGHT PT, KwOH CK: Randomized, Controlled Trial of - Compression Gloves in Rheumatoid Arthritis. Arthritis Care Res 1992, 5:223-228.

Two types of gloves were equally effective, but patients had specific preferences, suggesting that choice may result in improved compliance.

12. Hoenig H, Groff G, Pratt K, Goldberg E, Franck W: A Randomized Controlled Trial of Home Exercise on the Rheumatoid Hand. J Rbeumatol 1993, 20:785-789.

13. Deluhag B, Wollersjöl, Bjelue A: Effect of Active Hand Exercise and Wax Bath Treatment in Rheumatoid Arthritis Patients. Arthritis Care Res 1992, 5:87-92.

14. BRIGHTON SW, LUBbe JE, Van Der MerWE CA: The Effect of

-. a Long-Term Exercise Programme on the Rheumatoid Hand. Br J Rheumatol 1993, 32:392-395.
This controlled study suggests that a regular home exercise program and motivational reinforcement can increase and sustain grip and pincer grip strength over 4 years.

15. JOHNSON DP, EASTWOOD DM: Beneficial Effects of Continu- ous Passive Motion After Total Condylar Knee Arthroplasty. Ann $R$ Coll Surg Engl 1992, 74:412-416.

Compared with splinting, CPM reduced length of stay and improved ROM as measured after 1 year without negative effects to wound healing.

16. MCINNES J, LARSON MG, Daltroy LH, BROWN T, Fossel AH,

- Eaton HM, Shulman-Kirwan B, Steindorf S, Poss R, Liang

MH: A Controlled Evaluation of Continuous Passive Motion in Patients Undergoing Total Knee Arthroplasty. JAMA 1992, 268:1423-1428.

In addition to a standard rehabilitation program, CPM was effective in reducing the need for postoperative manipulations, which also resulted in lower overall rehabilitation costs. No increased frequency of wound healing problems was observed.

Gerold Stucki, MD, and Matthew H. Liang, MD, MPH, Brigham and Women's Hospital, Department of Rheumatology/Immunology, Multipurpose Arthritis Center, 75 Francis Street, Boston, MA 02115, USA. 